\title{
THE USE OF DOPPLER ULTRASONOGRAPHY FOR THE EVALUATION OF CEREBRAL ARTERY FLOW PATTERNS IN INFANTS WITH CONGENITAL HEART DISEASE
}

\author{
A. REBECCA SNIDER $\dagger$ \\ Department of Pediatrics, C. S. Mott Children's Hospital, University of Michigan Medical Center, \\ Ann Arbor, MI 48109, U.S.A.
}

\begin{abstract}
With the use of a two-dimensional range-gated pulsed-Doppler ultrasound system, Doppler examinations of the aorta and cerebral arteries were performed in 20 normal newborn infants and in 54 infants with various forms of congenital heart disease (i.e. patent ductus arteriosus, coarctation of the aorta, critical aortic stenosis, pulmonary atresia, truncus arteriosus). In the cerebral artery Doppler recordings of the normal infants, there was antegrade flow throughout systole and diastole. In the cerebral artery Doppler recordings of the infants with cardiac disease, various abnormal flow signals (i.e. retrograde or absent diastolic flow signals, increased systolic and diastolic flow signals, etc.) were found. Furthermore, the changes in the cerebral artery Doppler recordings were concomitant with changes in the aortic Doppler flow signals and the arterial blood pressure. Representative examples of the normal and abnormal Doppler flow patterns that were observed in the aorta and cerebral arteries are presented, and the mechanisms that could lead to these flow patterns are discussed.
\end{abstract}

Key Words: Doppler ultrasound, Cerebral blood flow, Congenital heart disease.

\section{INTRODUCTION}

In recent years, Doppler ultrasonography has been used extensively to examine the circulatory patterns of the brain in various disease states. Because of the limited access to the cerebrovascular circulation imposed by the bony skull of the adult, the Doppler technique has been used primarily to examine the flow patterns in the carotid and vertebral arteries extracranially (Strandness, 1978; Barnes and Wilson, 1975; Grossman and Wood, 1970). Other investigators have applied the Doppler probe to the exposed surface of the brain at the time of neurosurgery to locate and define deep-seated malformations in the brain (Nornes et al., 1979a, b). In the late 1970's, Bada and colleagues (1979) described a technique for recording intracranial flow patterns in newborn infants by placing the Doppler transducer over the anterior fontanel. Since then, abnormal cerebral artery Doppler flow patterns have been described in infants with a wide variety of neonatal disorders including asphyxia (Bashiru et al.,

† Please address all correspondence to A. Rebecca Snider, M.D., Associate Professor of Pediatrics (Cardiology), F 1609, C. S. Mott Children's Hospital, Ann Arbor, MI 48109.
1980), intraventricular hemorrhage (Bada et al., 1982; Perlman et al., 1983; Ahmann et al., 1983), infantile hydrocephalus (Hill and Volpe, 1982) and pneumothorax (Hill et al., 1982). In most instances, alterations in cerebral artery flow patterns were directly related to alterations in arterial blood pressure, a finding that suggests the occurrence of pressure-passive cerebral blood flow or impaired cerebral autoregulation in distressed newborn infants. The purpose of this report is to review the cerebral artery Doppler flow patterns in a group of infants with yet another cause for altered systemic blood pressure, that is, infants with various congenital cardiac defects.

\section{MATERIALS AND METHODS}

\section{Patients}

Fifty-four infants with various forms of congenital heart disease and 20 control infants underwent a Doppler ultrasound examination. The infants with congenital heart disease ranged in age from 1 day to 8 months and in weight from 0.70 to $3.8 \mathrm{~kg}$. Their cardiac diagnosis was based upon an evaluation by a pediatric cardiologist, which included a physical examination, electrocardiogram, chest roentgenogram 
and $\mathrm{M}$-mode and two-dimensional echocardiograms. In addition, 44 of the 54 infants in the congenital heart disease group underwent a complete cardiac catheterization. At the time of the Doppler examination, none of the infants in this group were acidotic, hypercarbic or extremely hypoxic by arterial blood gas determination.

By physical and echocardiographic examinations, the 20 control infants had no evidence of cardiac disease. The infants ranged in age from 1 to 7 days and in weight from 1.9 to $3.7 \mathrm{~kg}$.

\section{The Doppler examination}

To evaluate cerebral and aortic blood flow patterns, all infants were examined with a two-dimensional range-gated pulsed Doppler ultrasound system (Advanced Technology Laboratories). The Doppler recordings were made using a $3 \mathrm{MHz}$ medium focus transducer with a sample volume size of $2 \times 4 \mathrm{~mm}$. In the case of a few small premature infants in the patent ductus arteriosus group, a $5 \mathrm{MHz}$ medium focus transducer with a sample volume size of 1 $\times 1.5 \mathrm{~mm}$ at a focal depth of $3 \mathrm{~cm}$ was used.

With the aid of the two-dimensional echocardiographic display, the Doppler cursor line was placed through the vessel to be examined. The position of the sample volume along the cursor line was operatorcontrolled by a range-gating device and was indicated on the display by a crossbar. The echoes reflected from red blood cell flow within the region of the sample volume underwent fast Fourier transform analysis every $10 \mathrm{msec}$. The fast Fourier transform analysis divided the original complex Doppler-shifted wave-form into its frequency components and corresponding amplitudes. The resulting real-time spectral analysis was displayed on a video monitor with frequency shift on the vertical axis, time on the horizontal axis, and amplitude as shades of gray. In addition, blood flowing in a direction toward the transducer gave rise to Doppler signals that were displayed above the baseline, and blood flowing in a direction away from the transducer gave rise to Doppler signals displayed below the baseline.

The spectral tracings and a simultaneous electrocardiogram were recorded by a Tektronic fiber-optic recorder at paper speeds of 50 or $100 \mathrm{~mm} / \mathrm{sec}$. All tracings were recorded with a high-pass wall filter of $100 \mathrm{~Hz}$ and logarithmic amplification of the Doppler signal. At the end of each Doppler recording, a freezeframe of the two-dimensional echocardiogram showing the sample volume position was printed.

The Doppler examinations of the aorta and a basal cerebral artery were performed sequentially. For the aortic examination, the infant was lying supine with the head turned toward the left side. For the suprasternal long axis view of the aortic arch, the transducer was placed over the manubrium sternum with the plane of sound oriented between the right nipple and left scapular tip. Spectral tracings were then recorded from the descending aorta just above the diaphragm and, in some cases, from the ascending aorta.

In the control infants and in some of the infants with congenital heart disease (i.e., those with coarctation of the aorta and those with a large patent ductus arteriosus), flow patterns in the descending abdominal aorta were also examined using the subcostal sagittal view.

Cerebral artery Doppler recordings were obtained by placing the transducer on the anterior fontanel in a sagittal plane. Because of their prominent pulsations, the cerebral arteries were easy to locate. Spectral tracings were recorded from one or more cerebral arteries arising from the circle of Willis at the base of the brain. From the location on the two-dimensional echocardiographic display, these recordings were obtained from branches of the anterior or middle cerebral arteries. In order to obtain an adequate cerebral Doppler recording in some infants, it was necessary to tilt or rotate the transducer slightly from the sagittal plane.

\section{Doppler analysis}

The Doppler recordings of the control infants were compared to those of the infants with heart disease in order to detect qualitative differences in the waveforms (i.e. up slope, down slope, direction of diastolic flow). Also, values for the peak systolic frequency, trough diastolic frequency, acceleration time (time from the onset to the peak frequency of the systolic portion of the Doppler tracing) and the ratio of the acceleration time to the $R$ to $R$ interval were obtained by averaging the measurements from three to five consecutive complexes. In addition, the pulsatility index (PI) of the spectral tracings from the cerebral arteries was calculated according to the method of Bada et al. (1979):

$\mathrm{PI}=\frac{\text { peak systolic frequency }- \text { trough diastolic frequency }}{\text { peak systolic frequency }}$.

In the infants with retrograde or absent cerebral diastolic flow, the PI was assigned a value of 1.0.

The unpaired $t$ test was used for statistical comparisons of the control and patent ductus arteriosus groups and the control and coarctation group. The remaining diagnostic groups had too few patients for meaningful statistical analysis. 


\section{RESULTS}

\section{Control infants}

In all 20 normal newborn infants, the cerebral artery Doppler recordings (Fig. 1) showed antegrade flow throughout systole and diastole $(P I=0.73$ \pm 0.04 ; mean $\pm \mathrm{SD}$ ). In systole, the Doppler tracings had a rapid up slope to the peak systolic frequency followed by a gradual curvilinear decrease in frequency thoughout the remainder of systole and diastole. For the control group, the peak systolic frequency was $2.0 \pm 0.07 \mathrm{kHz}$; the trough diastolic frequency was $0.6 \pm 0.05 \mathrm{kHz}$; the acceleration time was $72 \pm 12$ msec; and the acceleration time per $\mathrm{R}$ to $\mathrm{R}$ interval was $0.12 \pm 0.025$.

In the normal newborn infants, the Doppler signals recorded from the descending thoracic aorta in systole were displayed below the baseline, indicating forward flow away from the transducer (Fig. 2). In early diastole, there was a small amount of retrograde flow associated with aortic valve closure, followed by no flow throughout the remainder of diastole. The systolic portion of the aortic Doppler tracing had a very rapid up slope and a very rapid down slope (acceleration time $=70 \pm 15 \mathrm{msec}$ and acceleration time per $\mathrm{R}$ to $\mathrm{R}=0.10 \pm 0.03$ ). From the suprasternal notch view, the Doppler beam was nearly parallel to flow in the descending aorta, and, therefore, the peak systolic frequency shifts were high.

The Doppler tracings from the descending abdominal aorta of the control infants were recorded from the subcostal sagittal view at angles ranging from $46^{\circ}$ to $68^{\circ}$. This resulted in smaller frequency shifts $(2.8 \pm 0.01 \mathrm{kHz})$ for comparable velocities (velocity $=149 \pm 35 \mathrm{~cm} / \mathrm{sec}$ ); however, the acceleration time $(64 \pm 16 \mathrm{msec})$ and the acceleration time per $R$ to $R$ interval $(0.097 \pm 0.02)$ were not significantly different from those recorded from the descending thoracic aorta in the suprasternal view.

\section{Patent ductus arteriosus}

Three infants with a clinically insignificant patent ductus arteriosus (PDA) and seven infants with a large patent ductus arteriosus were examined (Martin et al., 1982). In the three infants with a small PDA, the Doppler recordings from the descending aorta and cerebral arteries were identical to those of the normal infants. The PI of the Doppler recordings from the cerebral arteries of the small PDA infants was not statistically different from the PI of the cerebral arteries of the control infants.

In the seven infants with a large PDA, the Doppler recordings from the descending aorta (Fig. 3 ) showed normal forward flow down the descending aorta in systole and retrograde flow in diastole (large deflection above the baseline). The Doppler recordings from the cerebral arteries showed retrograde diastolic flow in three infants (Fig. 4), no recordable diastolic flow in one infant and markedly decreased diastolic flow in three infants. The retrograde flow signals were most prominent in mid- to late diastole. The systolic portion of the cerebral artery Doppler recordings had a rapid up slope, a rapid linear down slope, and a triangular shape. The PI of the Doppler recordings

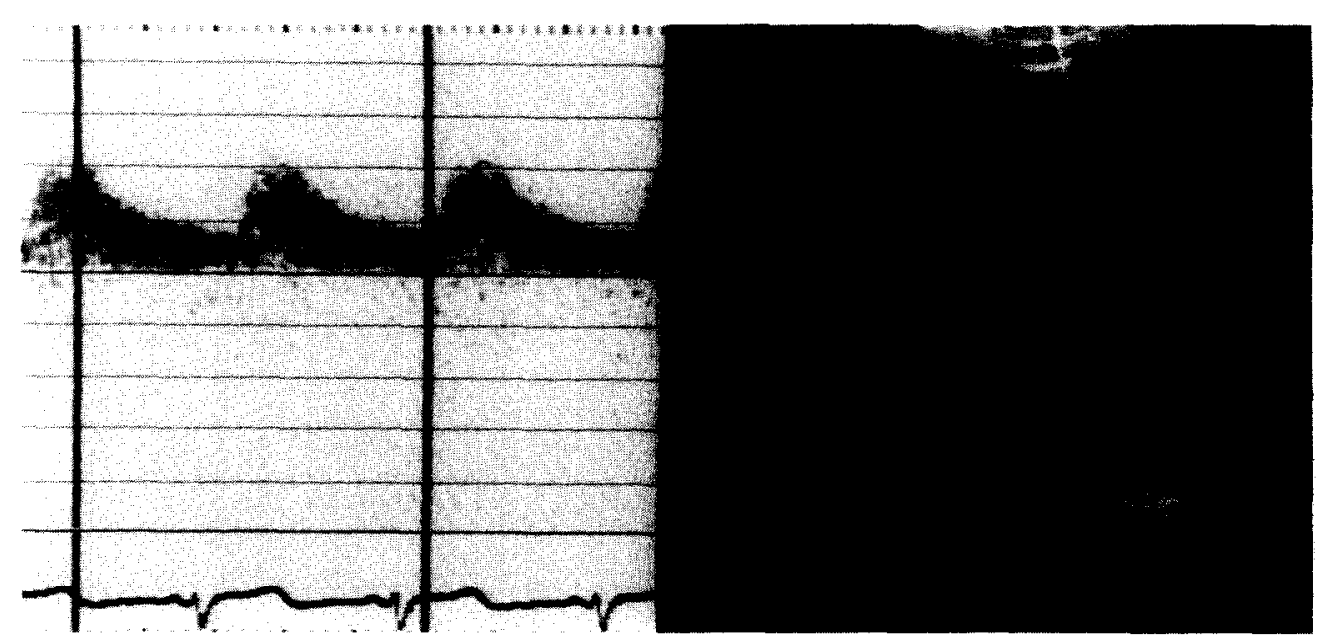

Fig. 1. Doppler spectral tracing (left) from a cerebral artery of a normal infant. To record the Doppler tracing, the transducer is placed on the anterior fontanel in a sagittal plane. The freeze-frame image of the sagittal view of the brain (right) shows the position of the sample volume (white arrow) when the spectral tracing was recorded. There was forward flow in the cerebral artery throughout systole and diastole resulting in Doppler signals that are displayed above the baseline. P, posterior; S, superior. Doppler calibration lines, $1 \mathrm{kHz}$. 


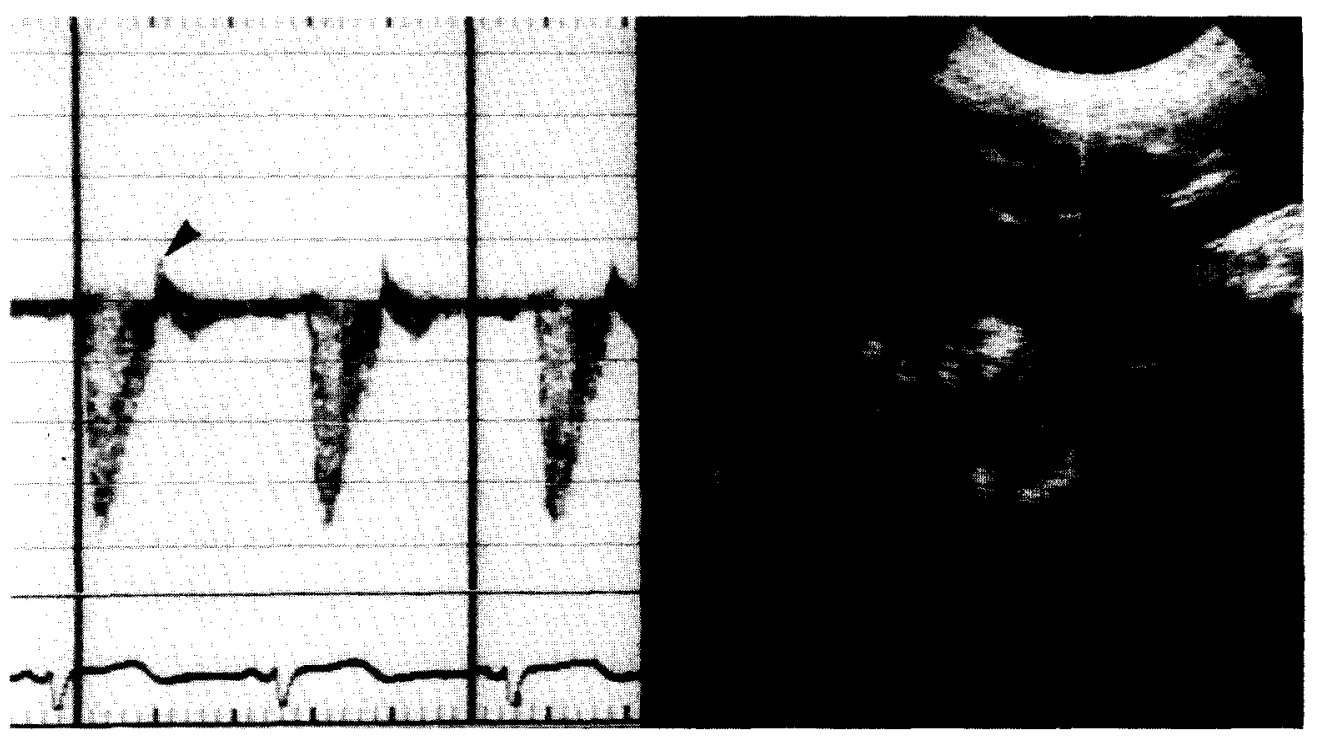

Fig. 2. Doppler spectral tracing (left) from the descending aorta (DAo) of a normal infant. The freeze-frame image of the suprasternal long axis view of the aortic arch (right) shows the position of the sample volume (crossbar on the cursor line) when the spectral tracing was recorded. Throughout systole, there was forward flow in the descending aorta away from the transducer resulting in Doppler signals that are displayed below the baseline. The signals in early diastole above the baseline (black arrow) are caused by the normal small amount of retrograde flow in the aorta associated with aortic valve closure. The spectral tracing for the remainder of diastole is on the baseline indicating no significant flow in the descending aorta throughout diastole. A, anterior;

AAo, ascending aorta; RPA, right pulmonary artery; S, superior. Doppler calibration lines, $1 \mathrm{kHz}$.

from the cerebral arteries of the large PDA infants $(0.96 \pm 0.06)$ was significantly elevated compared to the PI of normal infants.
After closure of the PDA in the seven infants with a large PDA, the Doppler recordings from the descending aorta and cerebral arteries were identical

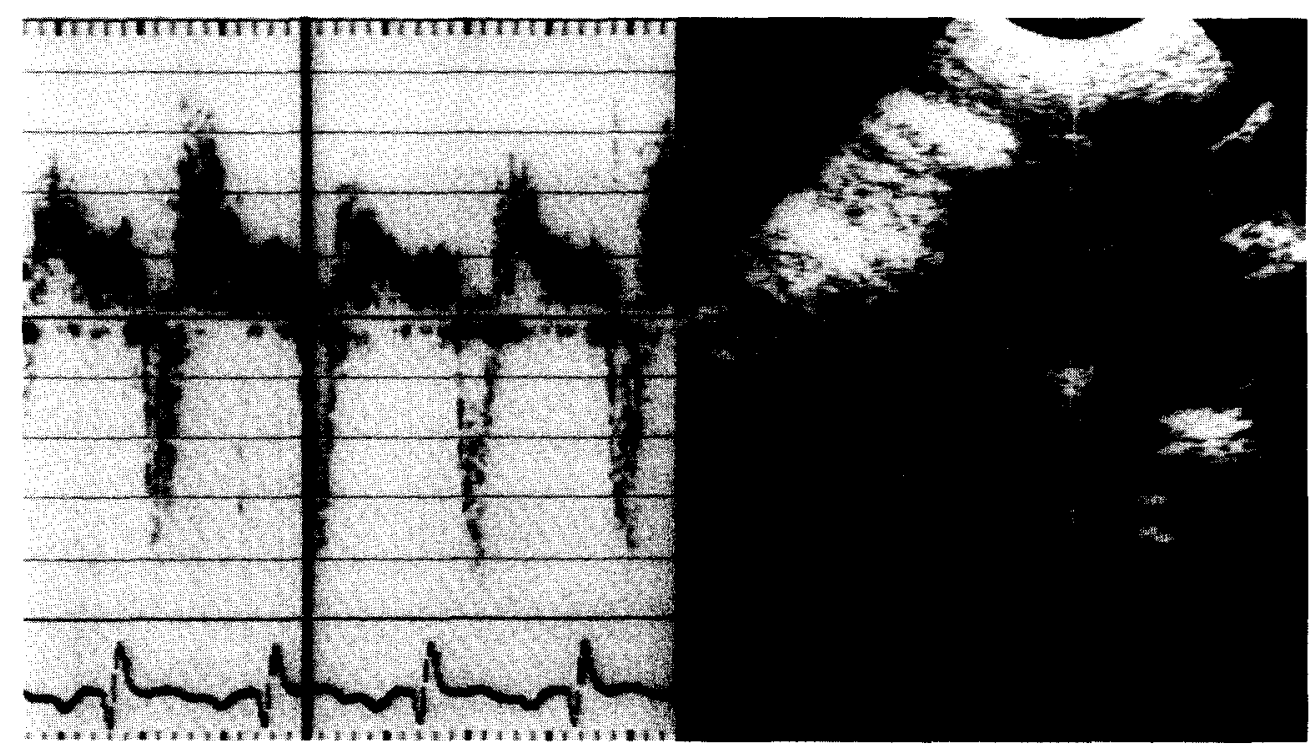

Fig. 3. Doppler spectral tracing (left) from the descending aorta (DAo) of an infant with a large patent ductus arteriosus. The freeze-frame image of the aortic arch on the right shows the position of the sample volume (white arrow) when the spectral tracing was recorded. In systole, there was forward flow down the descending aorta away from the transducer resulting in Doppler signals that are displayed below the baseline. Throughout diastole, there is a large deflection above the baseline indicating retrograde flow in the descending aorta toward the transducer. A, anterior; AAo, ascending aorta; S, superior. Doppler calibration lines, $1 \mathrm{kHz}$. 


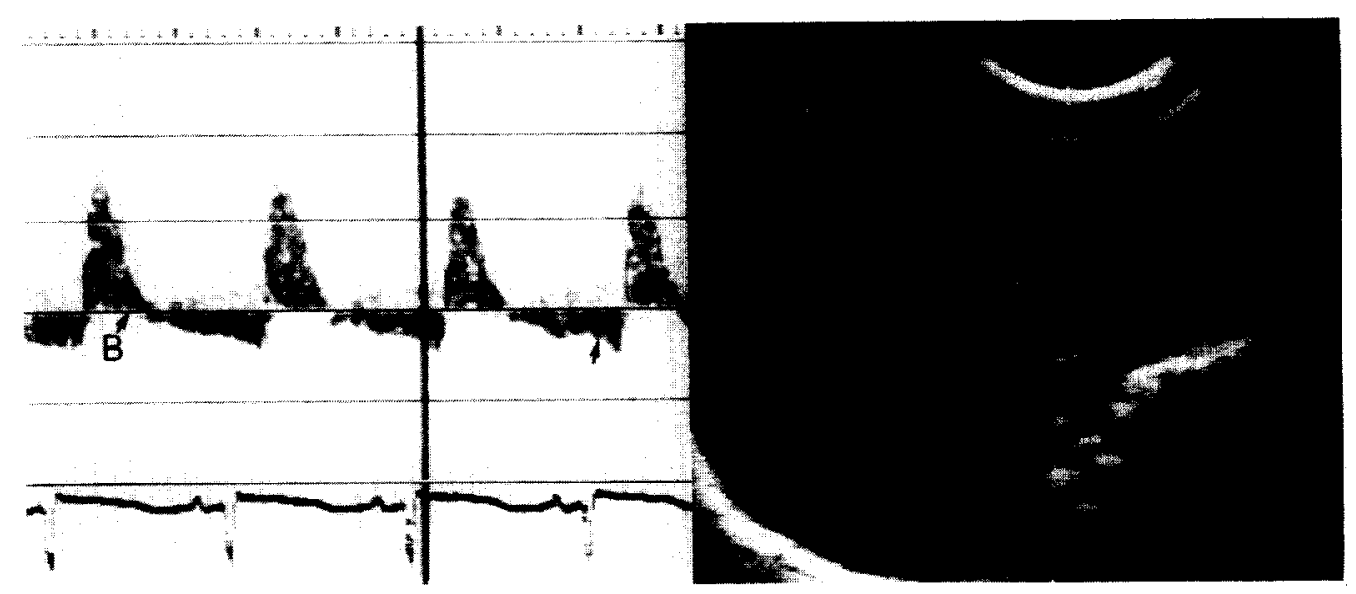

Fig. 4. Doppler spectral tracing (left) from a cerebral artery of an infant with a large patent ductus arteriosus. The freeze-frame image of the sagittal view of the brain (right) shows the pusition of the sample volume (white arrow) when the Doppler was recorded. The spectral tracing shows Doppler signals (black arrow) in mid- to late diastole below the baseline (B) indicating retrograde diastolic flow. In systole, the Doppler signals have a marked triangular shape, rapid up slope, and rapid down slope. A, anterior; LV, lateral ventricle; S, superior. Doppler calibration lines, $2 \mathrm{kHz}$. Figure reproduced with permission from Martin et al. (1982).

to those of the control group. In six infants after PDA closure, there were normal flow signals in the cerebral arteries throughout systole and diastole; however, in one infant, the cerebral artery flow signals remained low for $24 \mathrm{~h}$ after PDA closure (Fig. 5). The PI for the seven infants after PDA closure $(0.74$ \pm 0.04 ) was significantly different from the PI for the same seven infants before PDA closure and was not significantly different from the PI of the control group.

\section{Coarctation of the aorta}

Twenty-two infants with severe coarctation of the aorta and increased systemic arterial pressures proximal to the coarctation underwent Doppler examination (Snider and Howard, 1983). Doppler recordings from the aorta proximal to the coarctation (suprasternal notch view) showed a rapid up slope (acceleration time $=88 \pm 11 \mathrm{msec}$ and acceleration time per $R$ to $R$ interval $=0.18 \pm 0.038$ ), a rapid down slope, and a high peak systolic frequency (Fig. 6). The ascending aorta Doppler recordings often had evidence of systolic turbulence due to an associated abnormality of the left ventricular outflow tract (i.e. bicuspid aortic valve).

From the subcostal view, the Doppler recordings from the abdominal aorta distal to the coarctation showed a very slow up slope, a slow down slope, and a decreased peak systolic frequency (Fig. 7). The peak systolic frequency $(1.4 \pm 0.07 \mathrm{kHz})$, acceleration time (128 $\pm 3 \mathrm{msec}$ ), and acceleration time per $R$ to $R$ interval $(0.25 \pm 0.062)$ were significantly different

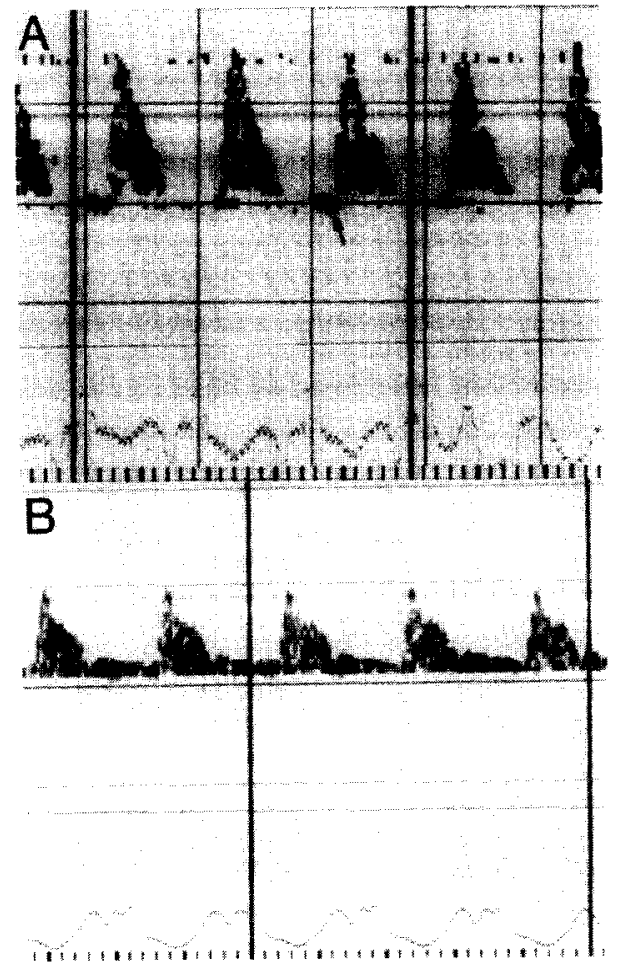

Fig. 5. (A) Doppler spectral tracing from a cerebral artery of an infant with a large patent ductus arteriosus. In this infant, there are Doppler signals in mid- to late diastole below the baseline (black arrow) indicating diastolic retrograde flow. The systolic portion of the Doppler recording has a triangular shape with a rapid up slope and a rapid, linear down slope. Doppler calibration lines, $1 \mathrm{kHz}$. (B) Doppler spectral tracing from a cerebral artery of the same infant following closure of the patent ductus arteriosus. The spectral tracing now shows forward flow throughout systole and diastole. Doppler calibration lines, $2 \mathrm{kHz}$. 


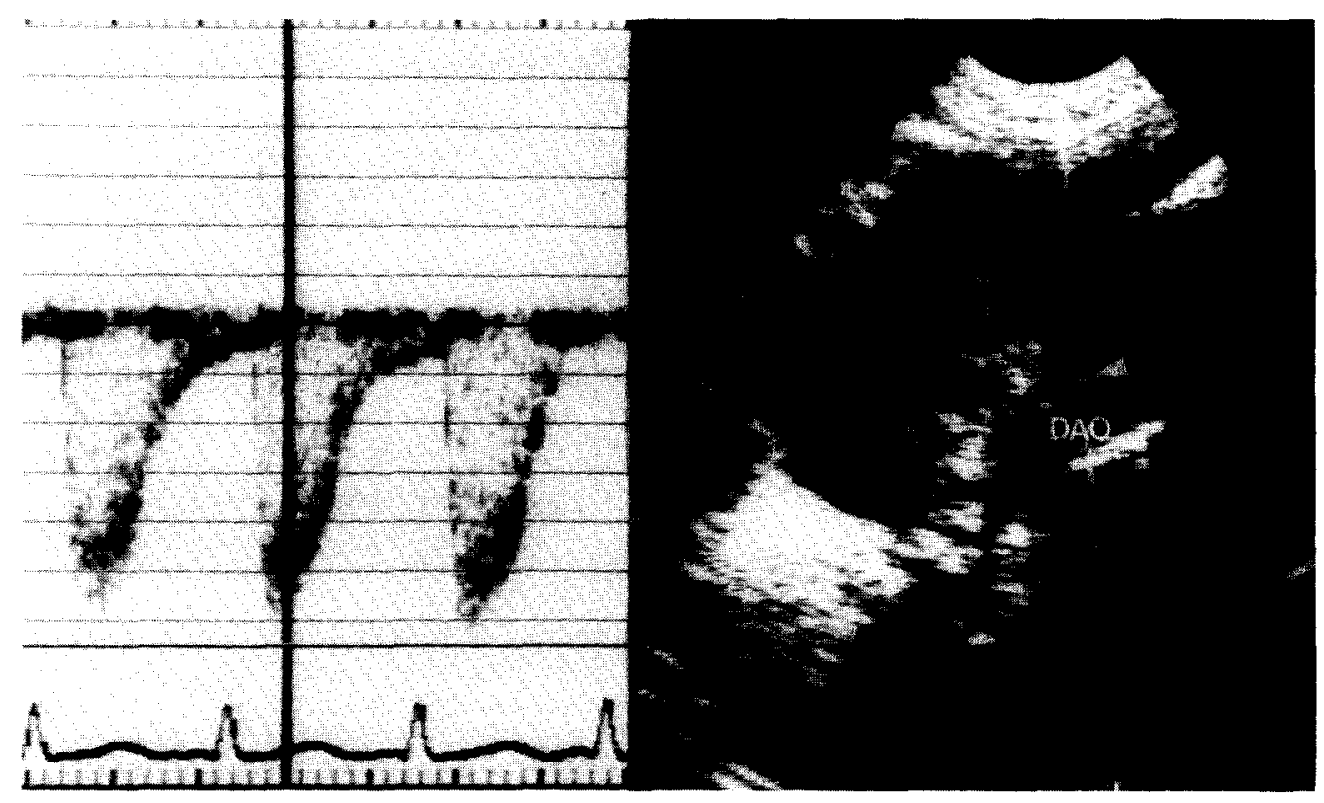

Fig. 6. Doppler spectral tracing (left) from the descending aorta (DAO) of an infant with a severe coarctation of the aorta. The freeze-frame image of the aortic arch (right) shows the position of the sample volume (white arrow) proximal to the coarctation when the Doppler signals were recorded. In systole, the spectral tracing shows a rapid acceleration time and a high peak systolic frequency. There is no significant flow in the aorta in diastole. A, anterior; AAO, ascending aorta; RPA, right pulmonary artery; S, superior. Doppler calibration lines, $1 \mathrm{kHz}$.

from those of the descending aorta Doppler proximal to the coarctation (suprasternal notch view, see above). The difference in peak systolic frequency was in part due to a loss of pulsatile flow in the descending aorta distal to the coarctation and in part due to different incidence angles of the ultrasound beam. From the

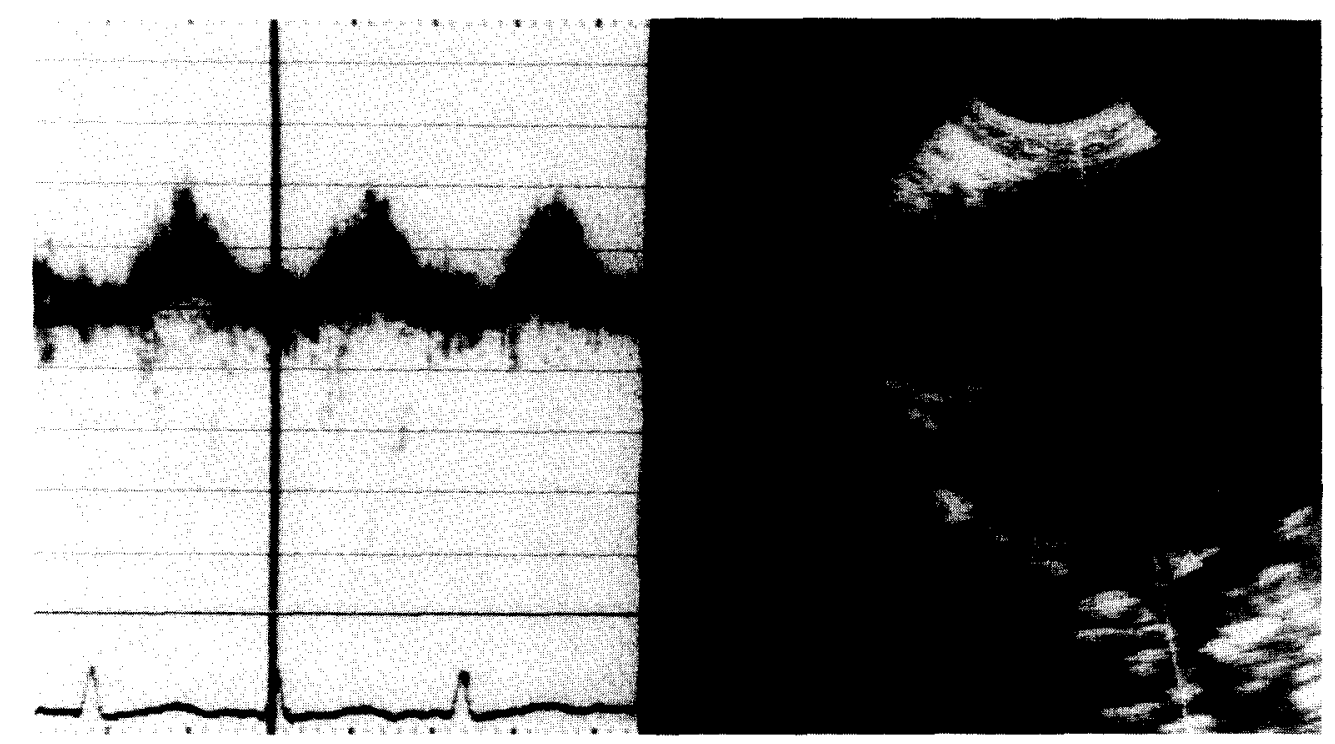

Fig. 7. Doppler spectral tracing (left) from the descending aorta (DAO) of the same infant as in Fig. 6. The freeze-frame image on the right shows a subcostal sagittal view of the abdominal aorta below the coarctation. The spectral tracing from the descending aorta shows a very slow up slope, a slow down slope, a low peak systolic frequency, and a prolonged acceleration time. This abnormal descending aorta Doppler tracing is probably caused by a loss of pulsatile flow in the descending aorta due to the presence of a severe obstruction in the aorta. A, anterior; $I$, inferior. Doppler calibration lines, $1 \mathrm{kHz}$. 
suprasternal notch view, the Doppler beam was nearly parallel to flow in the descending aorta, while, in the subcostal view, the angle between the Doppler beam and descending aorta varied between $50^{\circ}$ and $77^{\circ}$, resulting in smaller frequency shifts for the same velocities. However, the peak systolic frequency, acceleration time and acceleration time per $R$ to $R$ interval of the Doppler recordings from the descending abdominal aorta (subcostal view) of the coarctation infants were significantly different from those same parameters measured from the descending abdominal aorta (subcostal view) of normal infants at comparable angles $\left(46^{\circ}-68^{\circ}\right)$. Therefore, the difference in angles when recording the descending aorta from the subcostal view rather than from the suprasternal notch view did not account for most of the differences noted in the Doppler tracings from the descending abdominal aorta of the coarctation infants. In the infants who underwent surgical repair of the coarctation, the decreased peak systolic frequency and delayed acceleration time (slow up slope) in the descending abdominal aorta disappeared following removal of the aortic obstruction.

In the cerebral arteries of infants with a severe coarctation of the aorta, there was a slight decrease in the pulsatility index caused largely by an increase in the diastolic flow signals (Fig. 8). Athough, usually, the peak systolic frequency was increased, the trough diastolic frequency was increased to a far greater extent. For the cerebral arteries of the infants with coarctation, the PI was $0.69 \pm 0.04$, the peak systolic frequency was $3.2 \pm 0.08 \mathrm{kHz}$, and the trough diastolic frequency was $1.0 \pm 0.04 \mathrm{kHz}$. Following surgical repair of the coarctation and return of systemic arterial pressure to normal, peak systolic frequency, trough diastolic frequency and PI returned to normal (Fig. 9).

\section{Critical aortic stenosis}

In four newborn infants with critical valvar aortic stenosis (Fig. 10), the cerebral artery Doppler recordings showed a decreased peak systolic frequency $(1.4 \pm 0.1 \mathrm{kHz})$, a normal diastolic trough frequency $(0.5 \pm 0.04 \mathrm{kHz})$ and a decreased PI $(0.5 \pm 0.05)$. The cerebral Doppler recordings had a very slow up slope and a slow down slope. Each infant had a low aortic systolic blood pressure and a narrowed pulse pressure.

\section{Hypoplastic left heart syndrome}

In two infants with aortic atresia (hypoplastic left heart syndrome) and a patent ductus arteriosus, the cerebral artery Doppler recordings showed findings similar to those of infants with severe aortic stenosis (Fig. 11). The peak systolic frequency was markedly decreased, and the tracings showed a slow up slope or prolonged acceleration time. In both infants, the ductus arteriosus was patent, and descending aorta Doppler recordings showed retrograde diastolic flow. The cerebral Doppler recordings, likewise, showed

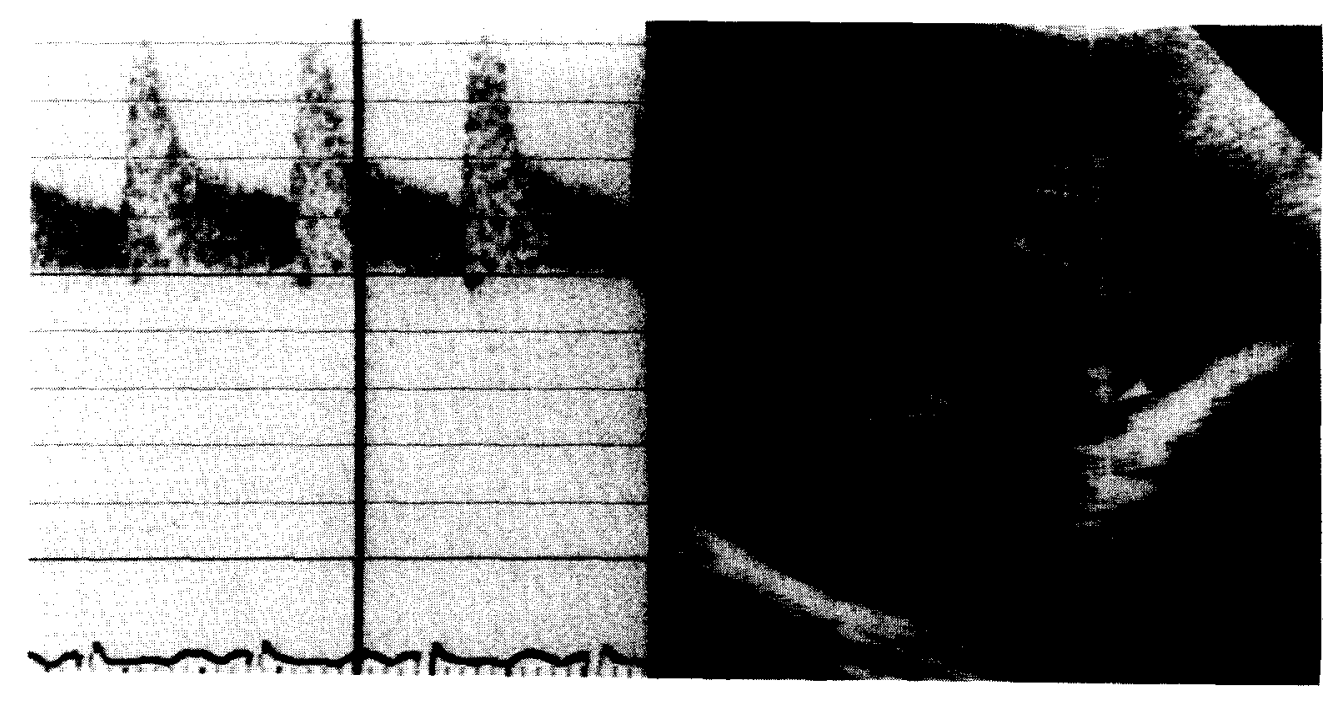

Fig. 8. Doppler spectral tracing (left) from a cerebral artery of an infant with a severe coarctation of the aorta. The freeze-frame image of the sagittal view of the brain (right) shows the position of the sample volume (white arrow) when the Doppler signals were recorded. The Doppler tracing shows an increase in peak systolic frequency and an increase in peak diastolic frequency. The increase in the flow signals is most likely caused by an increase in the aortic pressure proximal to the coarctation. $\mathrm{P}$, posterior; $\mathrm{S}$, superior. Doppler calibration lines, $1 \mathrm{kHz}$. 


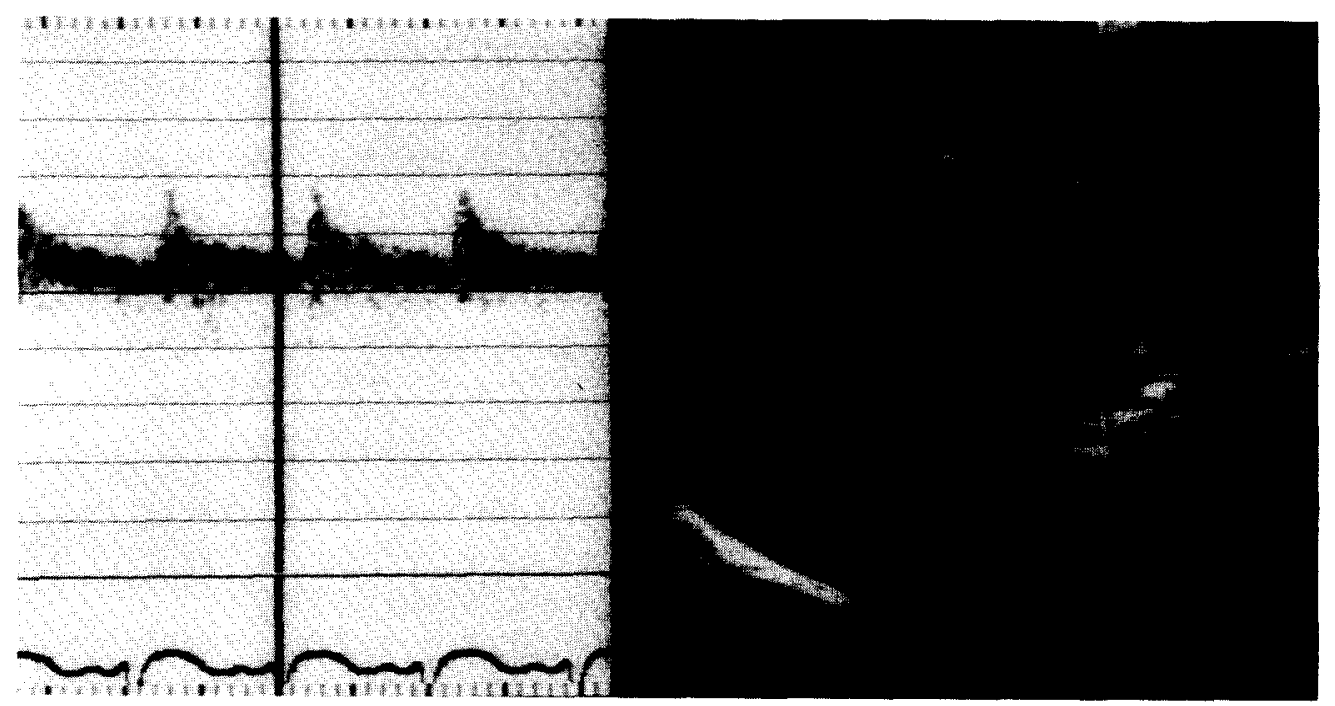

Fig. 9. Doppler spectral tracing (left) from a cerebral artery (right) of the same infant as in Fig. 8 following repair of coarctation of the aorta. The spectral tracing shows a marked decrease in the cerebral artery peak systolic frequency and a decrease in the peak diastolic frequency. The decrease in flow signals is most likely due to a decrease in aortic pressure following removal of the coarctation. $\mathrm{P}$, posterior; $\mathrm{S}$, superior. Doppler calibration lines, $1 \mathrm{kHz}$

decreased or retrograde diastolic flow. Both infants had low aortic systolic and diastolic blood pressures.

\section{Pulmonary atresia}

Two patterns of cerebral Doppler recordings were observed in infants with pulmonary atresia with or without a ventricular septal defect. In one group of four infants, both descending aorta and cerebral artery Doppler flow patterns appeared normal. At cardiac catheterization, these infants had small branch pulmonary arteries, no evidence of a patent ductus arteriosus and no significant systemic collateral vessels supplying the lungs.

In another group of nine infants with pulmonary atresia, the descending aorta Doppler recordings showed retrograde diastolic flow from the aorta to

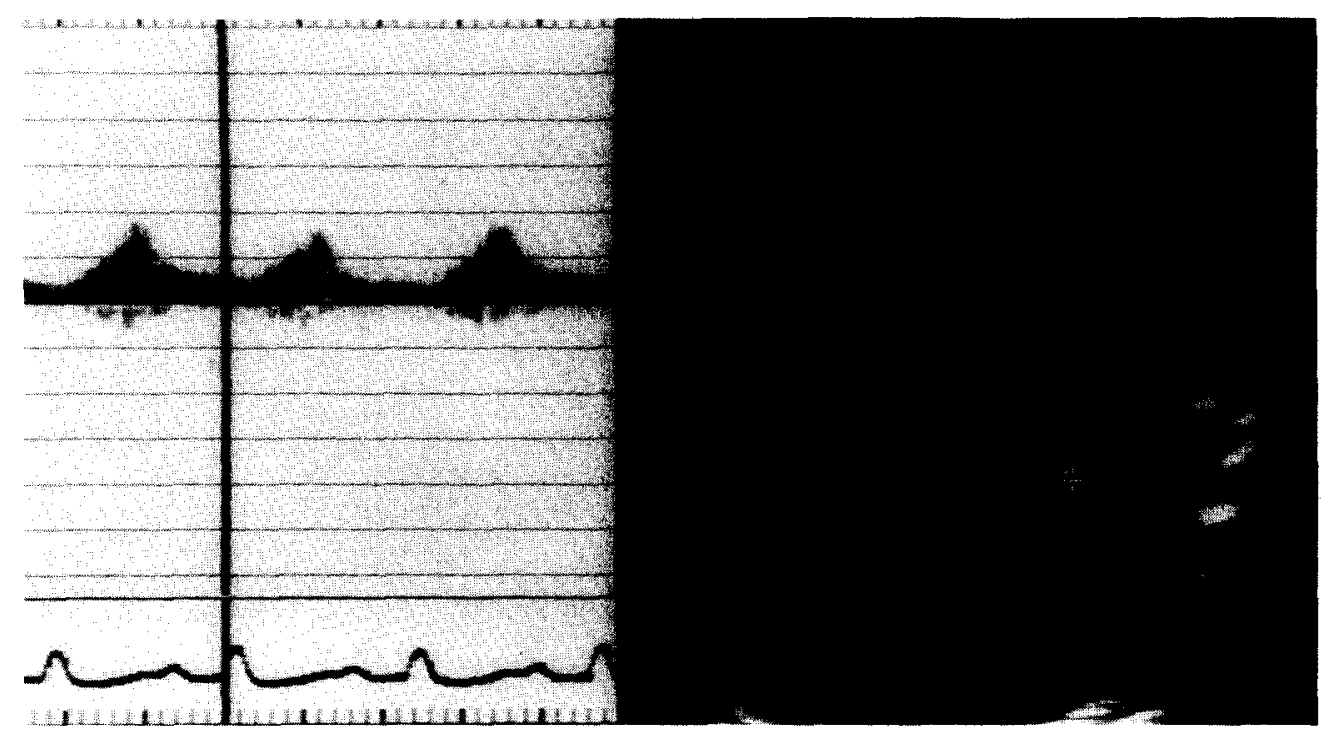

Fig. 10. Doppler spectral tracing (left) from a cerebral artery (right) of an infant with severe aortic stenosis. The Doppler tracing shows a marked decrease in peak systolic frequency, a decreased pulsatility index and a prolonged acceleration time. These findings are probably caused by diminished aortic systolic blood pressure and a narrowed aortic pulse pressure. $\mathbf{P}$, posterior; $\mathbf{S}$, superior. Doppler calibration lines, $1 \mathrm{kHz}$. 


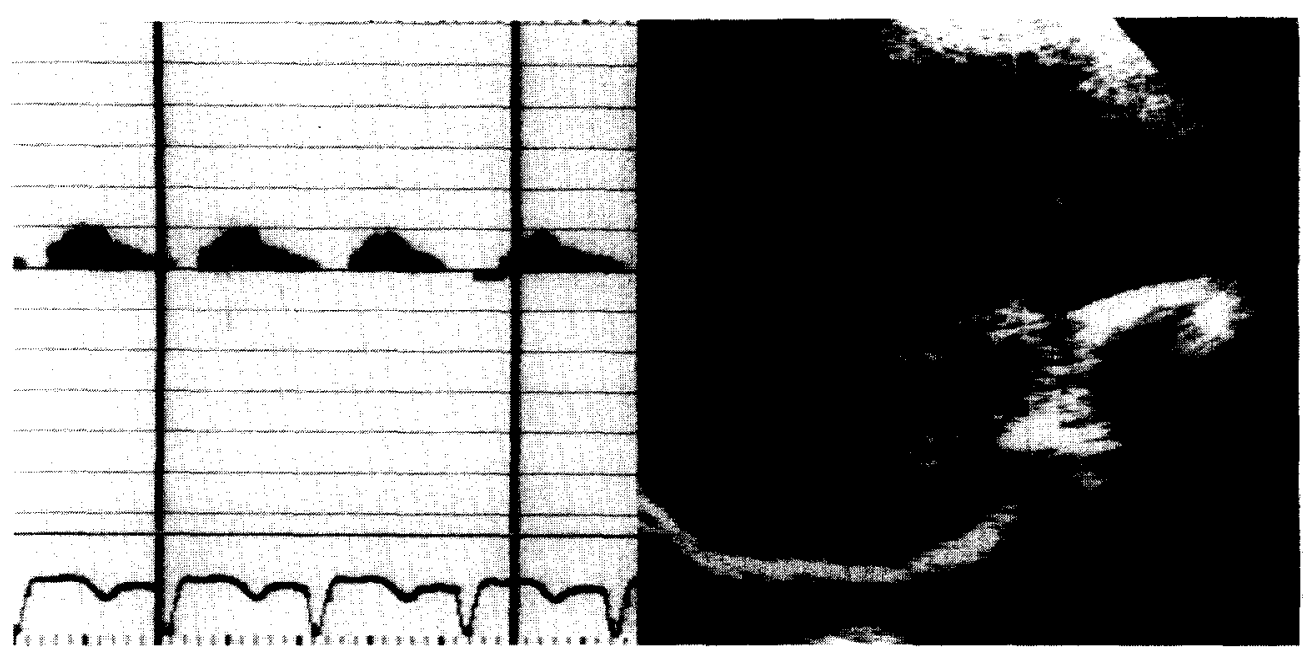

Fig. 11. Doppler spectral tracing (left) from a cerebral artery (right) of an infant with hypoplastic left heart syndrome and a patent ductus arteriosus. In systole, the Doppler tracing shows a markedly decreased peak systolic frequency and a prolonged acceleration time. In mid- to late diastole, there is absent forward flow in the cerebral artery. These findings are probably caused by the very low systemic arterial blood pressure in this infant and the presence of a large patent ductus arteriosus. $\mathrm{P}$, posterior; $\mathrm{S}$, superior. Doppler calibration lines, $1 \mathrm{kHz}$.

the pulmonary arteries. This was caused by the presence of either a patent ductus arteriosus or large systemic collateral vessels supplying the lungs. The cerebral artery Doppler recordings showed decreased or retrograde diastolic flow (Fig. 12), reflecting events in the systemic circulation.
Other cardiac defects with retrograde diastolic cerebral flow

Retrograde diastolic flow was observed in the cerebral arteries of infants with other forms of congenital heart disease. For example, this abnormal flow pattern was noted in five infants with truncus

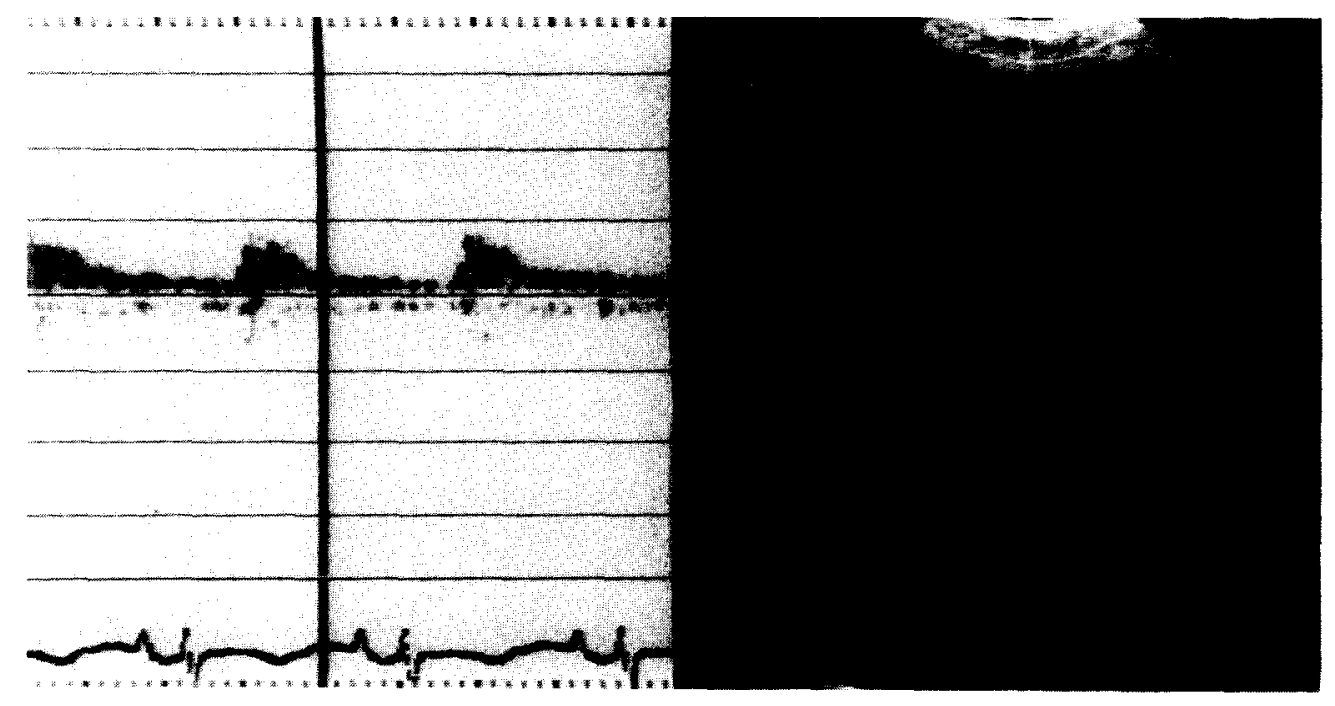

Fig. 12. Doppler spectral tracing (left) from the cerebral artery (right) of an infant with pulmonary atresia, intact ventricular septum, and patent ductus arteriosus. The spectral tracing shows continuous forward flow in the cerebral artery throughout systole and diastole; however, the diastolic flow signals are decreased and the pulsatility index is increased. This abnormal flow pattern is probably caused by the presence of a large left-toright shunt in diastole through the patent ductus arteriosus. The descending aorta Doppler recording in this infant showed retrograde diastolic flow. $\mathrm{P}$, postcrior; $\mathrm{S}$, superior. Doppler calibration lines, $1 \mathrm{kHz}$. 
arteriosus. In these infants, blood flowed from the aorta into the pulmonary arteries in systole and diastole. The descending aorta Doppler recording showed evidence of retrograde diastolic flow. On the cerebral artery Doppler recordings, the diastolic signals were either decreased, absent or retrograde.

In eight infants with complex cyanotic congenital heart disease and decreased pulmonary blood flow in whom a large aortopulmonary shunt had been surgically created, the descending aorta Doppler recording showed retrograde diastolic flow. Similarly, the Doppler recordings from the cerebral arteries showed retrograde diastolic flow (Fig. 13). This diastolic steal of blood from the cerebral arteries was probably caused by the large left-to-right shunt in systole and diastole through the surgical anastomosis.

\section{DISCUSSION}

The first group of patients with congenital heart disease whose cerebral artery flow patterns were studied with Doppler ultrasonography were preterm infants with a PDA. In the presence of a large PDA, the lower resistance pulmonary circulation communicates with the higher resistance systemic circulation resulting in a left-to-right shunting of blood from the aorta to the pulmonary arteries throughout systole and diastole. Several studies have shown that preterm infants with a large left-to-right ductal shunt are at a higher risk for developing necrotizing enterocolitis and intraventricular hemorrhage. Using a continuous wave Doppler ultrasound system, Serwer and colleagues $(1980,1982)$ showed retrograde diastolic flow in the descending aorta of infants with a large PDA. Retrograde diastolic flow disappeared following ligation of the ductus arteriosus. These authors postulated that retrograde diastolic flow in the descending aorta impaired circulation to the bowel and resulted in the development of necrotizing enterocolitis.

In order to determine whether diastolic shunting through the ductus arteriosus impaired circulation to the brain and contributed to the development of intraventricular hemorrhage in preterm infants, Perlman and colleagues (1981) examined the flow patterns in the anterior cerebral arteries of preterm infants with a PDA. Using a continuous wave Doppler flowmeter set to record advancing flow only, thesc investigators found a decrease in the cerebral artery diastolic flow signals and, therefore, an increase in PI. The increase in PI correlated with a decrease in arterial diastolic blood pressure, and both cerebral artery flow patterns and systemic arterial blood pressure returned to normal following closure of the PDA. These authors postulated that alterations in cerebral artery flow patterns caused by a large PDA may predispose the preterm infant either to ischemic or hemorrhagic brain injury. Using a continuous wave Doppler flowmeter set to record advancing flow on one channel and reverse flow on a second channel, Lipman and colleagues (1982) also noted a significant rise in the PI values during ductal patency. In addition, these investigators recorded retrograde diastolic flow

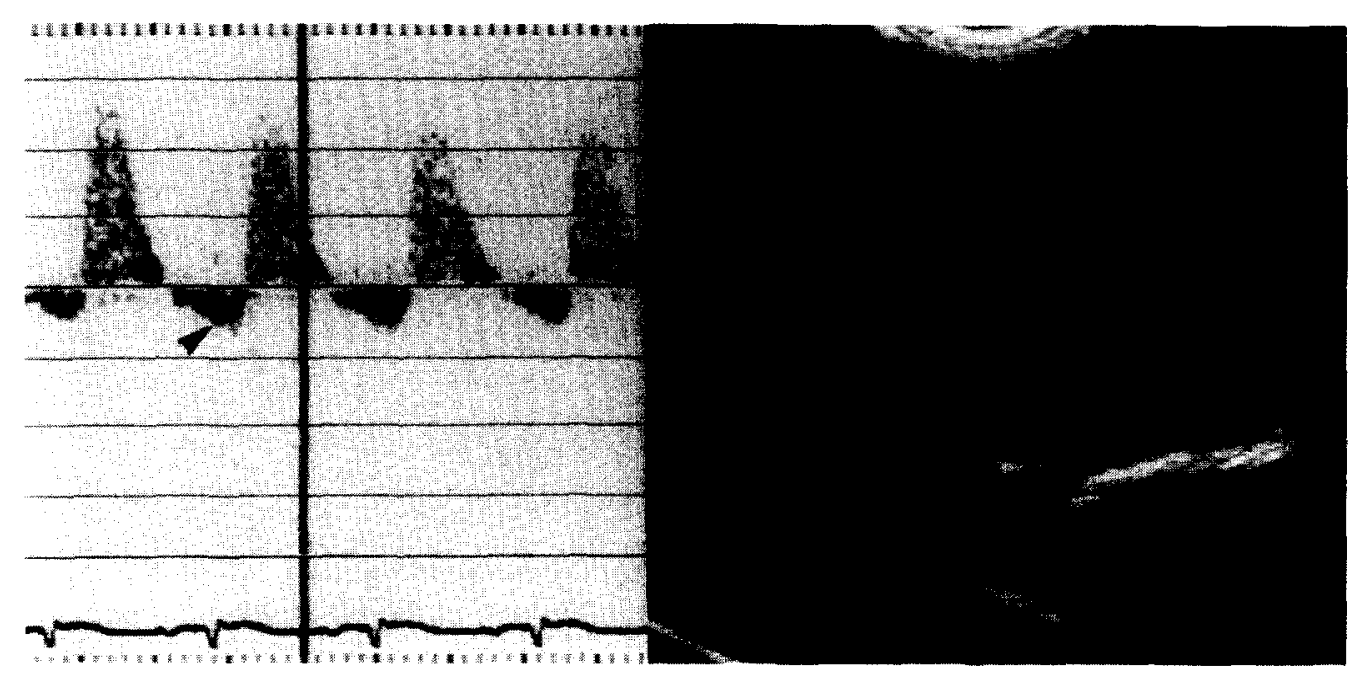

Fig. 13. Doppler spectral tracing (left) from a cerebral artery (right) of an infant with pulmonary atresia and a surgically-created aortopulmonary shunt. There is marked retrograde diastolic flow in the cerebral artery (black arrow). The retrograde diastolic flow in the cerebral artery may be caused by a diastolic steal of blood from the aorta and cerebral arteries by way of the large surgical anastomosis. The descending aorta Doppler recording in this infant showed retrograde diastolic flow. A, anterior; S, superior. Doppler calibration lines, $1 \mathrm{kHz}$. Figure reproduced with permission from Snider and Howard (1983). 
in the antcrior cercbral arterics of two infants. Following PDA closure, PI decreased to normal values in all infants.

In order to determine how alterations in systemic blood flow related to alterations in cerebral blood flow, Martin and associates (1982) used a range-gated pulsed Doppler ultrasound system (as noted in the Results section) to examine the aorta and cerebral arteries of preterm infants with a PDA. These investigators found that cerebral blood flow patterns were severely altered (retrograde or decreased diastolic flow signals, increased PI) in preterm infants with a large PDA and that cerebral blood flow patterns were abnormal only in those infants with concomitant retrograde diastolic flow in the descending aorta. This study introduced the use of two-dimensional pulsedDoppler ultrasonography for the purposes of imaging and recording the flow patterns in the cerebral arteries of infants through the anterior fontanel. Since then, this technique has been used (Snider and Howard, 1983) to examine the cerebral artery flow patterns in a group of infants with various congenital cardiac defects; and, in these cases, changes in cerebral blood flow patterns were associated with similar changes in arterial pressure and/or aortic blood flow patterns.

The use of the two-dimensional pulsed-Doppler ultrasound system provides several advantages in recording cerebral artery flow signals in infants. First, this system allows one to examine signals arising only from blood flowing in the vessel within the sample volume. The Doppler tracings obtained with the continuous wave system may contain contributions from blood flow in several vessels along the path of the beam; therefore, differences in the patterns of blood flow in different blood vessels might not be appreciated. Second, with this system, it is possible (even in small preterm infants) to obtain Doppler recordings from cerebral arteries that can be located precisely on the two-dimensional echocardiographic display, thus providing a method for examining blood flow patterns in specific areas of the brain. Finally, this technique allows one to combine a two-dimensional ultrasound study of the brain with a Doppler measurement of cerebral blood flow in one bedside examination.

Several mechanisms could explain the alterations in cerebral blood flow patterns found in infants with a large PDA or other cardiac defect with left-to-right diastolic shunting (i.e. truncus arteriosus). These changes could be caused by a true diastolic steal of blood flow from the cerebral arteries. With left-toright shunting of blood during diastole away from the aorta, retrograde flow of blood occurs in the descending aorta and there is a decrease in aortic diastolic blood pressure. As the shunt increases, diastolic flow in the cerebral arteries decreases and eventually reverses. Studies showing abnormal diastolic forward flow in the ascending aorta proximal to a large aortopulmonary communication lend support to the concept of a true diastolic steal of blood from the cerebral arteries (Wilcox et al., 1983; Spach et al., 1980; Rudolph et al., 1964). On the other hand, abnormal cerebral blood flow patterns could be caused by an increase in vascular impedance downstream from the cerebral artery sampled. Increased intracranial pressure, vasoconstriction of distal cerebral arteries and increased systemic venous pressure could increase the total cerebral vascular impedance. For example, an increase in systemic venous pressure caused by factors such as heart failure or total circulatory volume overload could impede venous drainage from the brain. With an increase in cerebral vascular impedance, total flow to the brain goes down; however, there is no true steal of blood from the brain. Regardless of the mechanism, the left-to-right diastolic shunt is the likely cause of the abnormal cerebral blood flow pattern since these patterns normalized after surgical closure of the shunt.

In infants with coarctation of the aorta and increased arterial blood pressure proximal to the obstruction, the cerebral artery Doppler signals were increased in systole and diastole. Following surgical repair of the coarctation, both cerebral artery flow patterns and arterial pressure returned to normal. Likewise, in infants whose cardiac defect causcd a low systemic blood flow and low arterial pressure (i.e. critical aortic stenosis, hypoplastic left heart), the cerebral artery Doppler signals were decreased in systole and diastole. The Doppler findings in these infants and in infants with a large left-to-right diastolic shunt support the concept of pressure-passive cerebral flow or impaired cerebral autoregulation in distressed neonates (Lou et al., 1979a-c). In normal patients, forward diastolic flow occurs in the anterior and middle cerebral arteries because of the low resistance of the cerebrovascular circulation. Therefore, alterations in the cerebral artery diastolic flow patterns can reflect either alterations in the systemic blood flow patterns or alterations in the distal resistance of the cerebral circulation. Changes in the distal resistance of the cerebral circulation can occur with changes in the arterial $\mathrm{pH}, \mathrm{pCO}_{2}$, or $\mathrm{pO}_{2}$. For example, acidosis and hypercarbia can cause cerebral vasodilatation and increased cerebral diastolic flow. The infants with congenital heart disease in this and other series did not have acidosis or hypercarbia; therefore, it appears that, over a wide range of arterial blood pressure, changes in cerebral artery flow patterns were 
caused by changes in systemic arterial flow patterns in these infants. The failure of the cerebral circulation to dilate or constrict and maintain normal forward diastolic flow velocities when systemic arterial diastolic pressure falls or rises implies either maximum cerebral vasodilation or vasoconstriction in these infants or pressure-passive cerebral blood flow.

The changes in cerebral artery diastolic flow patterns could predispose these infants to several types of cerebral injuries: (1) cerebral hyperperfusion could lead to rupture of thin-walled capillaries in the germinal matrix or subependymal regions of the brain (hemorrhagic injury); (2) diastolic steal of blood from the cerebral circulation could lead to underperfusion, structural damage and necrosis of brain cells especially in the "watershed" area of the brain (ischemic injury); and (3) fluctuations in flow patterns in the brain could lead to hemorrhagic injury (Perlman et al., 1983). Further studies are needed to correlate changes in cerebral blood flow patterns with tissue injury. Until these studies are completed, when caring for infants with congenital heart disease, we should be aware of the alterations in cerebral blood flow patterns that can occur with medical interventions (such as fluid restriction, administration or diuretics) with surgical interventions (such as placement of surgical shunts) or with the disease itself.

Acknowledgement-The author wishes to thank Mrs. Margaret Young for assistance in preparation of this manuscript.

\section{REFERENCES}

Ahmann P. A., Dykes F. D., Lazzara A., Holt P. J., Giddens D. P. and Carrigan T. A. (1983) Relationship between pressure passivity and subependymal/intraventricular hemorrhage as assessed by pulsed Doppler ultrasound. Pediatrics 72, 665-669.

Bada H. S., Hajjar W., Chua C. and Sumner D. S. (1979) Noninvasive diagnosis of neonatal asphyxia and intraventricular hemorrhage by Doppler ultrasound. J. Pediatr. 95, 775-779.

Bada H. S., Miller J. E., Menke J. A., Menten T. G., Bashiru M., Binstadt D., Sumner D. S. and Khanna N. N. (1982) Intracranial pressure and cerebral arterial pulsatile flow measurements in neonatal intraventricular hemorrhage. J. Pediatr. 100, 291-296.

Barnes R. W. and Wilson M. R. (1975) Doppler Ultrasonic Evaluation of Cerebrovascular Disease. University of Iowa Press, Iowa City.

Bashiru M., Russell J., Bada H. S., Menke J. A., Miles R. and Sumner D. (1980) Noninvasive detection of neonatal cerebrovascular disease. Bruit 4, 619-623.

Grossman B. L. and Wood E. H. (1970) Doppler ultrasonic evaluation of extracranial cerebrovascular disease. In Recent Advances in the Study of the Cerebral Circulation. (Edited by J. M. Tarases, H. Fischgold, D. Dilenge), pp. 175-187. Charles C. Thomas Publishers, Springfield.

Hill A. and Volpe J. J. (1982) Decrease in pulsatile flow in the anterior cercbral arterics in infantilc hydrocephalus. Pediatrics 69, 4-7.

Hill A., Perlman J. M. and Volpe J. J. (1982) Relationship of pneumothorax to occurrence of intraventricular hemorrhage in the premature newborn. Pediatrics 69, 144-149.

Lipman B., Serwer G. A. and Brazy J. E. (1982) Abnormal cerebral hemodynamics in preterm infants with patent ductus arteriosus. Pediatrics 69, 778-781.

Lou H. C., Lassen N. A. and Friis-Hansen B. (1979a) Impaired autoregulation of cerebral blood flow in the distressed newborn infant. J. Pediatr. 94, 118-121.

Lou H. C., Lassen N. A., Tweed W. A., Johnson G., Jones M. and Palahniuk R. J. (1979b) Pressure passive cerebral blood flow and breakdown of the blood-brain barrier in experimental fetal asphyxia. Acta Paediatr. Scand. 68, 57-63.

Lou H. C., Skov H. and Pedersen H. (1979c) Low cerebral blood flow: A risk factor in the neonate. J. Pediatr. 95, 606-609.

Martin C. G., Snider A. R., Katz S. M., Peabody J. L. and Brady J. P. (1982) Abnormal cerebral blood flow patterns in preterm infants with a large patent ductus arteriosus. J. Pediatr. 101, 587-593.

Nornes H., Grip A. and Wikeby P. (1979a) Intraoperative evaluation of cerebral hemodynamics using directional Doppler technique. Part 1: Arteriovenous malformations. $J$. Neurosurg. 50, 145 151

Nornes H., Grip A. and Wikeby P. (1979b) Intraoperative evaluation of cerebral hemodynamics using directional Doppler technique. Part 2: Saccular aneurysms. J. Neurosurg. 50, 570-577.

Perlman J. M., Hill A. and Volpe J. J. (1981) The effect of patent ductus arteriosus on flow velocity in the anterior cerebral arteries: Ductal steal in the premature newborn infant. $J$. Pediatr. 99, 767-771.

Perlman J. M., McMenamin J. B. and Volpe J. J. (1983) Fluctuating cerebral blood-flow velocity in respiratory-distress syndrome: Relation to the development of intraventricular hemorrhage. N. Engl. J. Med. 309, 204-209.

Rudolph A. M., Scarpelli E. M., Golinko R. J. and Gootman N. L. (1964) Hemodynamic basis for clinical manifestations of patent ductus arteriosus. Am. Heart J. 68, 447-452.

Serwer G. A., Armstrong B. E. and Anderson P. A. W. (1980) Noninvasive detection of retrograde descending aortic flow in infants using continuous wave Doppler ultrasonography. $J$. Pediatr. 97, 394-400.

Serwer G. A., Armstrong B. E., Sterba R. J. and Anderson P. A. W. (1981) Alterations in carotid arterial velocity-time profile produced by the Blalock-Taussig shunt. Circulation 63, 1115-1120.

Serwer G. A., Armstrong B. E. and Anderson P. A. W. (1982) Continuous wave Doppler ultrasonographic quantitation of patent ductus arteriosus flow. J. Pediatr. 100, 297-301.

Snider A. R. and Howard E. A. (1983) The evaluation of cerebral artery flow patterns with Doppler ultrasonography. In Pulsed Doppler Ultrasound in Clinical Pediatrics (Edited by W. Berman Jr.), pp. 93-140. Futura Publishing Co., New York.

Spach M. S., Serwer G. A., Anderson P. A. W., Canent R. V. Jr. and Levin A. R. (1980) Pulsatile aortopulmonary pressure-flow dynamics of patent ductus arteriosus in patients with various hemodynamic states. Circulation 61, 110-122.

Strandness D. E. Jr. (1978) The use of ultrasound in the evaluation of peripheral vascular disease. Prog. Cardiovasc. Dis. 20, 403422 .

Wilcox W. D., Carrigan T. A., Dooley K. J., Giddens D. P., Dykes F. D., Lazzara A., Ray J. L. and Ahmann P. A. (1983) Rangegated pulsed Doppler ultrasonographic evaluation of carotid arterial blood flow in small preterm infants with patent ductus arteriosus. J. Pediatr. 102, 292-302. 